\title{
Early and Rapid Targeting of Eye-Specific Axonal Projections to the Dorsal Lateral Geniculate Nucleus in the Fetal Macaque
}

\author{
Andrew D. Huberman, ${ }^{1}$ Colette Dehay, ${ }^{4}$ Michel Berland, ${ }^{3,4}$ Leo M. Chalupa, ${ }^{1,2}$ and Henry Kennedy ${ }^{4}$ \\ ${ }^{1}$ Center for Neuroscience and ${ }^{2}$ Section of Neurobiology, Physiology, and Behavior and Department of Ophthalmology, University of California, Davis, Davis, \\ California 95616, and ${ }^{3}$ Hopital Lyon-Sud and ${ }^{4}$ Institut National de la Santé et de la Recherche Médicale U371, Cerveau et Vision, Department of Cognitive \\ Neurosciences, PrimaBio, Institut Fédératif des Neurosciences de Lyon, Université Claude Bernard Lyon 1, 69500 Bron, France
}

The emergence of eye-specific axonal projections to the dorsal lateral geniculate nucleus (dLGN) is a well established model system for exploring the mechanisms underlying afferent targeting during development. Using modern tract tracing methods, we examined the development of this feature in the macaque, an Old World Primate with a visual system similar to that of humans. Cholera toxin $\beta$ fragment conjugated to Alexa 488 was injected into the vitreous of one eye, and СТ $\beta$ conjugated to Alexa 594 into the other eye of embryos at known gestational ages. On embryonic day 69 (E69), which is $\sim 100 \mathrm{~d}$ before birth, inputs from the two eyes were extensively intermingled in the dLGN. However, even at this early age, portions of the dLGN were preferentially innervated by the right or left eye, and segregation is complete within the dorsalmost layers 5 and 6. By E78, eye-specific segregation is clearly established throughout the parvocellular division of the dLGN, and substantial ocular segregation is present in the magnocellular division. By E84, segregation of left and right eye axons is essentially complete, and the six eye-specific domains that characterize the mature macaque dLGN are clearly discernable. These findings reveal that targeting of eye-specific axonal projections in the macaque occurs much earlier and more rapidly than previously reported. This segregation process is completed before the reported onset of ganglion cell axon loss and retino-dLGN synapse elimination, suggesting that, in the primate, eye-specific targeting occurs independent of traditional forms of synaptic plasticity.

Key words: eye-specific; retinogeniculate; visual system; primate; dLGN; axon guidance

\section{Introduction}

In mammals, projections from the two eyes to the dorsal lateral geniculate nucleus (dLGN) are segregated (Jones, 1985). Rakic (1976) first examined the development of this feature using monocular injections of radioactive proline to label ganglion cell axons in fetal macaque embryos. In the youngest fetus, injected on embryonic day 64 (E64) and killed on E78, proline-labeled axons appeared to fill the entire dLGN. In contrast, in the fetus injected on E110 and killed on E124, labeled axons occupied restricted

\footnotetext{
Received 0ct. 14, 2004; revised March 8, 2005; accepted March 11, 2005

This work was supported by National Institutes of Health Grants EY11369 (Dr. Barbara Chapman kindly provided support for A.D.H.), EY015387 (Vision Science training grant/J. Werner Chair provided support for A.D.H.), EY03991 (L.M.C.), and P20 MH6095 (National Institute of Mental Health and National Science Foundation) (L.M.C.); National Eye Institute Grant EY12576 (L.M.C.); and a Research to Prevent Blindness award (L.M.C.). Support was also obtained from Institut National de la Santé et de la Recherche Médicale, French Ministère de la Recherche Grants ACI0220582 (C.D.) and ACI 03568 (H.K.), European Community Grant QLG3-2000-00158 (C.D.), and Biomed Grant BMH4 (T961604 (H.K.). We thank Pascale Giroud and Véronique Cortay for excellent technical assistance and Fabrice Macaire for animal care.

Correspondence should be addressed to any of the following: Leo M. Chalupa, Section of Neurobiology, Physiology, and Behavior, Department of Ophthalmology, and Center for Neuroscience, University of California, Davis, Davis, CA 95616, E-mail: Imchalupa@ucdavis.edu; Henry Kennedy, Institut National de la Santé et de la Recherche Medicale U371, Cerveau et Vision, Department of Cognitive Neurosciences, PrimaBio, Institut Fédératif des Neurosciences de Lyon, Université Claude Bernard Lyon 1,69500 Bron, France, E-mail:kennedy@lyon.inserm.fr; or Andrew D. Huberman, Department of Neurobiology, Sherman Fairchild Building, Stanford University School of Medicine, Stanford, CA 94305, E-mail: adh1@stanford.edu.

D01:10.1523/JNEUROSCI.4292-04.2005

Copyright $\odot 2005$ Society for Neuroscience $\quad$ 0270-6474/05/254014-10\$15.00/0
}

regions of the dLGN. A third fetus was injected on E130 and killed on E144; labeled axons occupied restricted regions of the dLGN and were mirror symmetric and complimentary in the two hemispheres. These findings suggested that eye-specific segregation emerges from a state in which axons from the two eyes are initially intermingled throughout the dLGN. The segregation process appeared to occur between E78 and E124, with additional refinements in eye-specific projections occurring until E144. Subsequent studies examined the development of eye-specific retinogeniculate projections in other species (hamster, So et al., 1978; ferret, Linden et al., 1981; cat, Shatz, 1983; mouse, Godement et al., 1984; rat, Jeffrey, 1984), using both radioactive and nonradioactive tracers to label ganglion cell axons. These studies confirmed Rakic's main conclusion that eye-specific retinogeniculate projections emerge from a state in which axons from the two eyes overlap. However, the mechanisms that underlie development of eye-specific retinogeniculate projections still remain controversial (for review, see Chalupa and Huberman, 2004; Grubb and Thompson, 2004).

The long gestation of the macaque provides a unique opportunity to temporally dissociate the various cellular events that have been implicated in the formation of eye-specific projections. Furthermore, much is known about the major developmental events in the embryonic macaque visual system, including the timing of retinal ganglion cell and dLGN cell birth and matura- 
tion (Rakic, 1977a; Shatz and Rakic, 1981; La Vail et al., 1991), the attrition of optic nerve axons (Rakic and Riley, 1983), the ingrowth and elaboration of retinogeniculate axons (Meissirel et al., 1997; Snider et al., 1999), and synapse formation in the dLGN (Hendrickson and Rakic, 1977). Because Rakic's study relied on tracers now known to be prone to "spillover" (LeVay et al., 1978; Ruthazer et al., 1999; Crair et al., 2001) and long postinjection survival periods, a precise delineation of when eye segregation occurs in this important model species is lacking.

Here we explore the development of eye-specific retinogeniculate connections in the macaque, using modern, highresolution tracing techniques not prone to spillover. Our results show that segregation occurs much earlier and more rapidly in prenatal development than previously recognized. The revised timing of eye-specific segregation reported here argues that, contrary to what has been hypothesized previously, axon arbor pruning, ganglion cell death, and synapse elimination are unlikely to account for the formation of eye-specific retinogeniculate projections.

\section{Materials and Methods}

Animals. All procedures were performed according to National Institutes of Health guidelines and in strict compliance with institutional protocols of Institut National de la Santé et de la Recherche Médicale (Lyon, France), Inspection des Services Vétérinaires (Lyon, France), European directive 86/609, and the California Regional Primate Center at University of California, Davis. Injections were performed on six macaque fetuses (E68, E72, E77, E77, E83, and E96). Successful labeling from both eyes was obtained in the fetuses injected on E68, E77 $(n=2)$, and E83. In the E72 and E96 fetuses, the projections from only one eye were successfully labeled; the labeling pattern in the dLGN of these animals was in agreement with the conclusions regarding overlap versus segregation obtained from the other animals, but, for obvious reasons, tissue from these two animals could not be used for visualization or quantification of binocular overlap in dLGN. Quantification was performed from serial sections of the entire dLGN in both hemispheres of the animals in which dual-eye labeling was successful.

Timed breeding of macaque monkeys. Experiments were performed on cynomolgus monkeys (Macaca fascicularis) maintained on $12 \mathrm{~h}$ light/ dark cycles. To obtain timed-pregnant monkeys, "optimal" breeding days were determined by (1) monitoring the average length of a given animal's menstrual cycle over 6 consecutive months, (2) dividing this average cycle length by 2 , and (3) subtracting $2 \mathrm{~d}$ from this value. Animals were paired with a breeder male from $2 \mathrm{~d}$ before until $2 \mathrm{~d}$ after this optimal breeding day. This allows the gestational age of the fetus to be determined with an accuracy of $\pm 2 \mathrm{~d}$. E1 represents the $24 \mathrm{~h}$ after mating. The mean duration of gestation for M. fascilcularis (used here) and $M$. mulatta (the species used by Rakic) is $165 \mathrm{~d}$. The ranges of gestation are reported to vary somewhat between these two species (Roonwal and Mohnot, 1977). However, we observed similar timetables and tempos of prenatal visual system development in both species (Rakic, 1974; Dehay et al., 1993). Therefore, we are confident that our results can be directly compared across these two species.

In utero surgery. The pregnant monkey was premedicated with atropine $(0.2 \mathrm{mg} / \mathrm{kg}$, i.m. ) and prepared for surgery under ketamine hydrochloride $(10 \mathrm{mg} / \mathrm{kg})$, chlorpromazine $(1 \mathrm{mg} / \mathrm{kg}$, i.m.), and salbutamol sulfate $(0.125 \mathrm{mg}$, i.m.). After intubation, anesthesia was continued with halothane in an $\mathrm{N}_{2} \mathrm{O} / \mathrm{O}_{2}$ mixture (70:30). Throughout the procedure, the heart rate was monitored and artificial respiration was adjusted to maintain the end-tidal $\mathrm{CO}_{2}$ at $4.5-6 \%$. Core body temperature was monitored rectally and maintained at $37^{\circ} \mathrm{C}$. A ventral midline abdominal incision allowed exposure of the uterus. The fetal head was located by palpation and maneuvered to a nonplacental location at which uterotomy was performed. The pregnant monkey received postoperative medication consisting of a muscle relaxant (salbutamol sulfate, $0.125 \mathrm{mg}$, i.m.) twice daily and an analgesic (tiemonium methylsulfate, $0.4 \mathrm{mg} / \mathrm{kg}$ ).
Intravitreal injections of anterograde tracers. To label retinogeniculate projections, cholera toxin $\beta$ fragment (CT $\beta$ ) conjugated to Alexa 488 dye (CT $\beta-488)$ (green; $0.5 \%$ dissolved in sterile saline; Molecular Probes, Eugene, OR) was injected into the vitreal chamber of one eye, and CT $\beta$ conjugated to Alexa 594 dye (CT $\beta$-594) (red; $0.5 \%$ dissolved in sterile saline) was injected into the vitreal chamber of the other eye, using a sterile, short-beveled, 30 gauge needle (product 328438; Becton Dickinson, Franklin Lakes, NJ). Injections were made along the margin of the corneo-scleral junction, anterior to the neural retina. A "stop" was placed on the needle shaft to prevent the needle tip from progressing past the center of the vitreal chamber. After the needle was inserted, $10-15 \mu \mathrm{l}$ of CT $\beta$ conjugate was infused into the vitreal chamber, and the needle was removed. The optimal location of the needle stop and the volume of tracer for each age were determined from macaque eyes harvested in previous studies. CT $\beta$ conjugates bind to GM1 gangliosides, which are highly enriched in retinal ganglion cell axons, are not prone to spillover, are rapidly transported $(>4 \mathrm{~mm} / \mathrm{hr})$, and do not exhibit any biological activity (Rathjen and Gierer, 1981; Wu et al., 1999; Huberman et al., 2002; Muscat et al., 2003). After allowing $24 \mathrm{~h}$ for tracer transport, cesarean section was performed according to previously described protocols (Smart et al., 2002). The embryo was killed by an overdose of sodium pentobarbital and perfused transcardially with physiological saline, followed by $4 \%$ paraformaldehyde, and the brain was removed, postfixed for $8 \mathrm{~h}$, and then cryoprotected in 30\% sucrose, 70\% PBS solution for $48 \mathrm{~h}$.

Tissue processing and imaging. The brain was frozen on dry ice and sectioned coronally at $50 \mu \mathrm{m}$ on a freezing microtome. Sections were mounted onto gelatin-coated slides and coverslipped with Vectashield mounting media (Vector Laboratories, Burlingame, CA). Slides were viewed with a $3900 \times 3600$ pixel Zeiss (Thornwood, NY) Axiocam digital camera mounted on a Zeiss Axioplan microscope. Images of every section spanning the rostrocaudal extent of each dLGN were acquired using a $5 \times$ Zeiss PlanNeofluar objective. These images were then montaged together to produce the whole-dLGN photomicrographs. Highermagnification images of selected regions of the dLGN were acquired using Zeiss $10 \times$ and $20 \times$ Neofluar objectives. Confocal images were acquired using an Olympus Optical (Tokyo, Japan) FV500 confocal microscope and a $40 \times$ oil immersion objective. Details regarding optical section thickness are described in the figure legends. Coverslips were then removed, and the tissue was rinsed, dehydrated, and thionin stained to reveal cytoarchitecture.

Image analysis. Raw images of the dLGN were imported to Photoshop (Adobe Systems, San Jose, CA) and then set at a threshold 30\% above background (a non-retinorecipient portion of the tissue slice $1 \mathrm{~mm}$ lateral to the midline of the thalamus). The $30 \%$ value is based on previous studies (Huh et al., 2000; Huberman et al., 2002, 2003; Stellwagen and Shatz, 2002) and evaluation of signal-to-noise in tissue from animals at different ages. Threshold images were set to black $(<30 \%$ above threshold) or white ( $>30 \%$ above threshold). Measurements of the area of the dLGN occupied by the projections of each eye were calculated by automatically selecting all white pixels within the image frame (Image; Scion, Frederick, MD). Measurements of overlap were calculated by multiplying the threshold image of the contralateral eye inputs to the dLGN, with the threshold image of the ipsilateral eye inputs to the same dLGN. In the resulting image, white pixels correspond to locations in which the contralateral and ipsilateral afferents were both present; overlap area was calculated by automatically selecting all of the white pixels within the image frame and dividing that value by the total area of the dLGN in that same tissue section (using Scion Image software). Every tissue section from both dLGNs was included in this analysis. The sample size for each age is included in the legend for Figure 7. Because of a lack of other benchmarks at the ages studied here (such as the optic disk representation), rostral, middle, and caudal were distinguished on the basis of the size and morphology of the neighboring ventral LGN. This evenly divided the dLGN into thirds across its rostrocaudal extent (supplemental Figs. 1-3, available at www.jneurosci.org as supplemental material). 

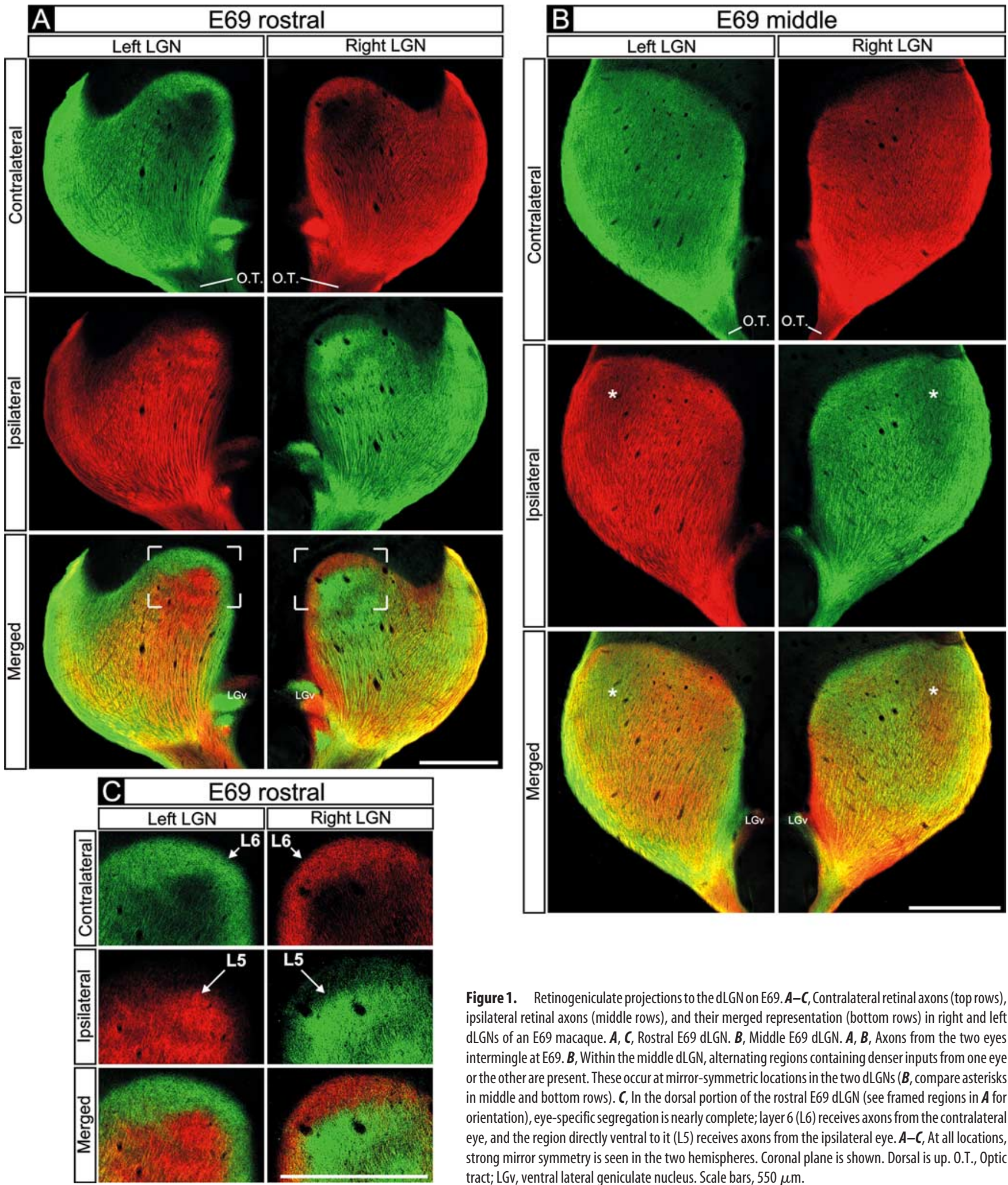

Figure 1. Retinogeniculate projections to the dLGN on E69.A-C, Contralateral retinal axons (top rows), ipsilateral retinal axons (middle rows), and their merged representation (bottom rows) in right and left dLGNs of an E69 macaque. $\boldsymbol{A}, \boldsymbol{C}$, Rostral E69 dLGN. $\boldsymbol{B}$, Middle E69 dLGN. $\boldsymbol{A}, \boldsymbol{B}$, Axons from the two eyes intermingle at $E 69$. $\boldsymbol{B}$, Within the middle $\mathrm{dLGN}$, alternating regions containing denser inputs from one eye or the other are present. These occur at mirror-symmetric locations in the two dLGNs ( $\boldsymbol{B}$, compare asterisks in middle and bottom rows). $C$, In the dorsal portion of the rostral E69 dLGN (see framed regions in $\boldsymbol{A}$ for orientation), eye-specific segregation is nearly complete; layer 6 (L6) receives axons from the contralateral eye, and the region directly ventral to it (L5) receives axons from the ipsilateral eye. $A-C$, At all locations, strong mirror symmetry is seen in the two hemispheres. Coronal plane is shown. Dorsal is up. 0.T., Optic tract; LGv, ventral lateral geniculate nucleus. Scale bars, $550 \mu \mathrm{m}$.

\section{Results}

Binocular projections to the $\mathrm{E} 69$ macaque dLGN

To determine when eye-specific retinogeniculate projections emerge during development, we injected a macaque fetus on E68, the earliest age when the full cohort of axons from the two eyes has arrived in the dLGN (Meissirel et al., 1997), and then killed the fetus on E69. Axons from the two eyes extensively overlap through most of the E69 dLGN (Fig. 1A,B). Each third of the dLGN along the rostrocaudal extent is hereafter referred to as rostral, middle, or caudal (see Materials and Methods) (supplemental Figs. 1-3, available at www.jneurosci.org as supplemental material). In the middle and caudal E69 dLGN, binocular overlap is extensive throughout the nucleus (Fig. $1 B$ ). However, even in the region of overlap, the density of label from the two eyes varies 

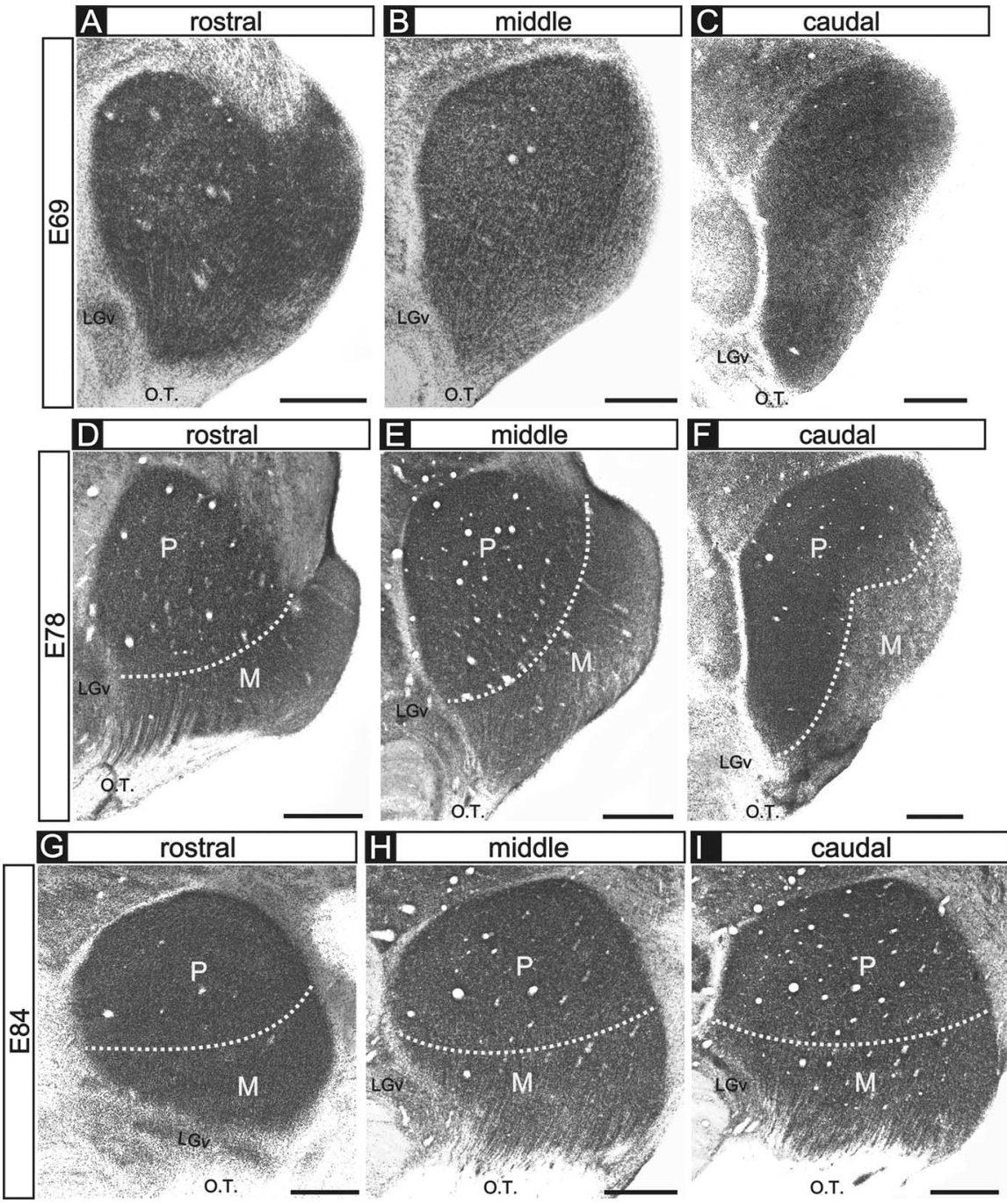

Figure 2. Cytoarchitectural differentiation of the dLGN. $A-I$, Photomicrographs of thionin-stained tissue sections of the embryonic macaque dLGN at E69 (A-C), E78 (D-F), and E84 (G-I). Rostral $(\boldsymbol{A}, \boldsymbol{D}, \boldsymbol{G})$, middle $(\boldsymbol{B}, \boldsymbol{E}, \boldsymbol{H})$, and caudal $(\boldsymbol{C}, \boldsymbol{F}, \boldsymbol{I})$ levels are shown for each age. At the ages shown here, there are no intralaminar spaces corresponding to eye-specific cellular layers, consistent with previous reports (Rakic, 1977a). $\boldsymbol{A}-\boldsymbol{C}$, At E69, the M and P divisions cannot reliably be distinguished at all rostrocaudal locations. However, in the E78 $(\boldsymbol{D}-\boldsymbol{F})$ and the E84 $(\mathbf{G}-\boldsymbol{I})$ specimens, the $\mathrm{M}$ and $\mathrm{P}$ divisions can be broadly distinguished on the basis of cell spacing and morphology of the dLGN at all rostral, middle, and caudal locations. Dotted lines indicate the boundaries between the M and P divisions. Coronal plane is shown. Dorsal is up, and medial is to the left. 0.T., Optic tract; LGv, ventral lateral geniculate nucleus. Scale bars, $500 \mu \mathrm{m}$.

in a complementary manner; regions containing denser label from one eye receive less dense projections from the opposite eye. These variations are mirror symmetric in the two dLGNs (Fig. $1 \mathrm{~B}$, asterisks), indicating that they were not caused by damage to the retina or regional differences in dye uptake/intensity. Hence, although substantial intermingling of axons from the two eyes is present at E69, regions of the $\mathrm{dLGN}$ already receive axons predominantly from one eye or the other. Furthermore, in the dorsal portion of the rostral E69 dLGN (Fig. 1 $A, C$ ), eye-specific segregation is virtually complete; the dorsalmost region (layer 6) contains axons almost exclusively from the contralateral eye, whereas the region just ventral to layer 6 (layer 5) receives axons almost exclusively from the ipsilateral eye (Fig. $1 \mathrm{~A}$, boxed areas in bottom row, $C$ ), as is the case in the adult macaque.

\section{Eye-specific magnocellular and parvocellular differentiation of the dLGN}

In carnivores and primates, eye specificity in the dLGN is characterized by two major features: (1) the nonoverlapping termina- tion zones formed by axons from the two eyes, and (2) layers of dLGN neurons that mirror the eye-specific termination zones and are separated from one another by cell-sparse intralaminar regions (for review, see Jones, 1985). Evidence from a variety of species indicates that eye-specific cellular layers develop subsequently to the segregation of retinal afferents from the two eyes (macaque, Rakic, 1977a,b; ferret, Linden et al., 1981; tree shrew, BrunsoBechtold and Casagrande, 1982; cat, Shatz, 1983). The emergence of eye-specific cellular layers in the dLGN is mediated by factors involving the presence and segregation of retinal axons (Brunso-Bechtold and Casagrande, 1985; Casagrande and Condo, 1988a), although nonretinal factors contribute as well (for review, see Casagrande and Condo, 1988b). In the present report, we focused on the segregation of retinal axons from the two eyes. At the ages we studied (E69-E84), cellular layers are not present at any location in the dLGN (Fig. 2A-I), consistent with previous reports in the macaque that cellular layers emerge in the dLGN from E90 onward (E90+) (Rakic, 1977a,b).

The adult macaque dLGN is also divided into magnocellular (M) and parvocellular $(\mathrm{P})$ divisions, which relay motion and form/color visual information, respectively (Livingstone and Hubel, 1988). During development, the macaque dLGN undergoes substantial rotation between E50 and E90 (Rakic, 1977a; Meissirel et al., 1997), making it difficult to precisely delineate the $\mathrm{M}$ and $\mathrm{P}$ divisions at the ages we studied here. Indeed at E69, M and P divisions are not reliably discernable at all rostrocaudal locations (Fig. 2A-C). However, by E78 and thereafter, the $M$ versus $P$ regions can be broadly distinguished at any location along the rostrocaudal extent of the dLGN on the basis of their position, morphological features, and regional differences in cell density (Fig. 2D-F) (Rakic, 1977a; Meissirel et al., 1997), allowing us to assess the emergence of eye-specific segregation in these two functional subdivisions of the dLGN.

\section{Retinogeniculate projections in the E78 macaque}

Examination of the pattern of binocular retinogeniculate projections in embryos injected with CT $\beta$ tracers on E77 and killed on E78 revealed that, within the P division of the E78 dLGN, axons from the two eyes are segregated along the full rostrocaudal extent of the nucleus and exhibited striking mirror symmetry in the two hemispheres (Fig. 3A,B) (supplemental Fig. 2, available at www.jneurosci.org as supplemental material). Within some areas of the $\mathrm{M}$ division, axons from the two eyes still appeared overlapping. In the rostral E78 dLGN, eye-specific segregation within the $\mathrm{M}$ division is also pronounced (Fig. $3 A$, Merged). Overlap is more apparent in the $\mathrm{M}$ division of the middle and caudal dLGN (Fig. 


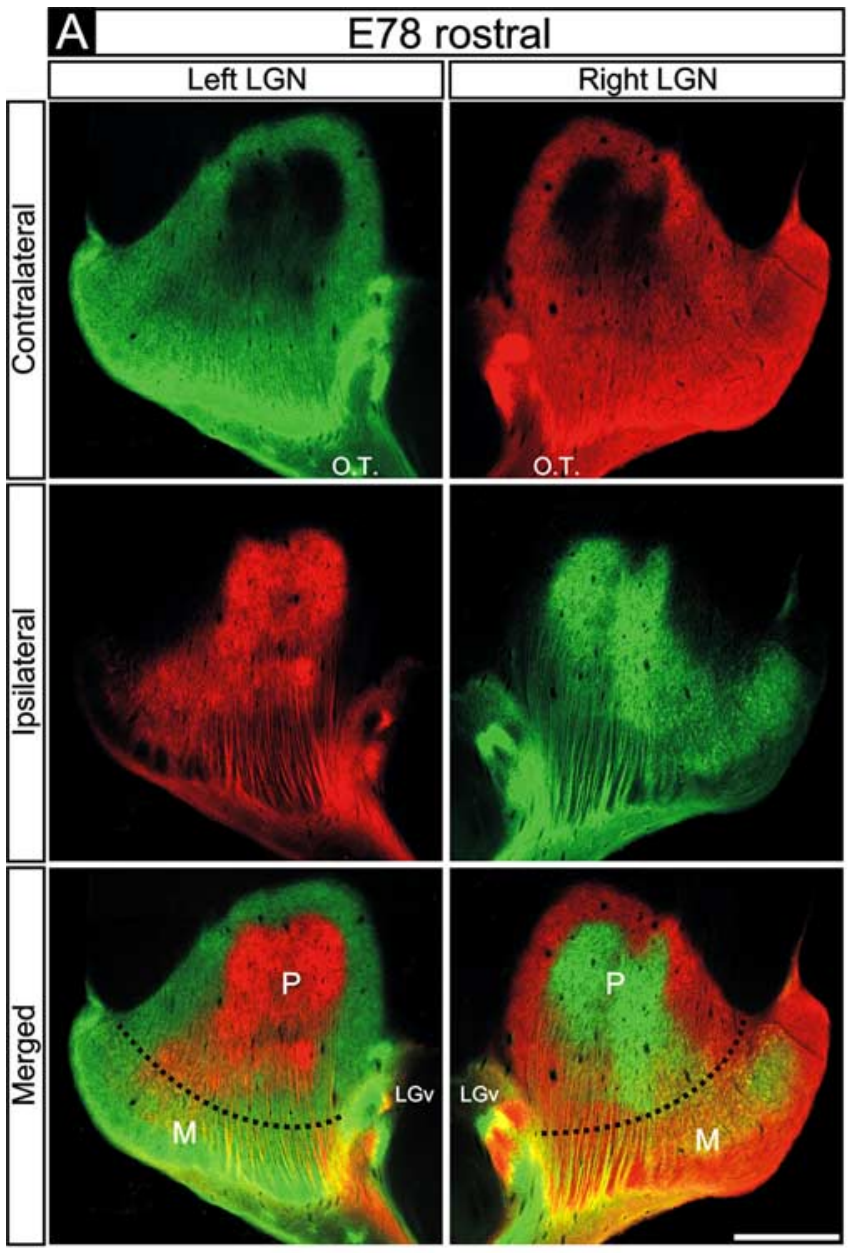

Figure 3. Retinogeniculate projections to the $\mathrm{dLGN}$ on E78. $\boldsymbol{A}$, $\boldsymbol{B}$, Contralateral retinal axon (top rows), ipsilateral retinal axons (middle rows), and their merged representation (bottom rows) in the right and left dLGNs of an E78 macaque. $\boldsymbol{A}$, Rostral dLGN; $\boldsymbol{B}$, middle dLGN. $\boldsymbol{A}, \boldsymbol{B}$, Eye-specific segregation is present throughout the P division of the E78 $\mathrm{dLGN}$. $\boldsymbol{B}$, In the middle $\mathrm{dLGN}$, some overlap is seen in the M division. (However, for discussion of the contribution of fibers of passage to the overlap observed, see Results.) Even in the middle M division, however, substantial eye-specific segregation is present; only contralateral eye axons are found within the ventrolateral $M$ division (asterisks), which corresponds to layer 1 . Boundary between the $M$ and $P$ division is indicated by the dotted lines in Merged panels. $\boldsymbol{A}-\boldsymbol{D}$, Coronal plane is shown. Dorsal is up. 0.T., Optic tract; LGv, ventral lateral geniculate nucleus. Scale bars, $550 \mu \mathrm{m}$.

$3 B$, Merged). Even in these portions of the $\mathrm{M}$ division, however, layer 1 (at this stage located in the ventrolateral dLGN) exclusively receives contralateral eye axons (Fig. $3 B$, asterisks), as is the case in the adult. CT $\beta$ tracing thus revealed that at E78, the agewhen radioactive tracers indicated the presence of complete binocular overlap in the dLGN (Rakic, 1976), eye-specific segregation is complete throughout the P division of the dLGN and is essentially complete in much of the $\mathrm{M}$ division. It is likely that the relatively greater overlap seen in some portions of the M division is attributable to fibers of passage, although actual timing differences of eye-specific segregation in the $\mathrm{M}$ versus $\mathrm{P}$ zones could also exist (see Discussion).

\section{Assessing the degree of right and left eye axon intermingling in "overlap" zones}

A handful of in vitro physiological studies have been performed that indicate that, at stages of development when binocular overlap is present in the dLGN, there is functional convergence of right and left eye axons onto single dLGN neurons (Shatz and

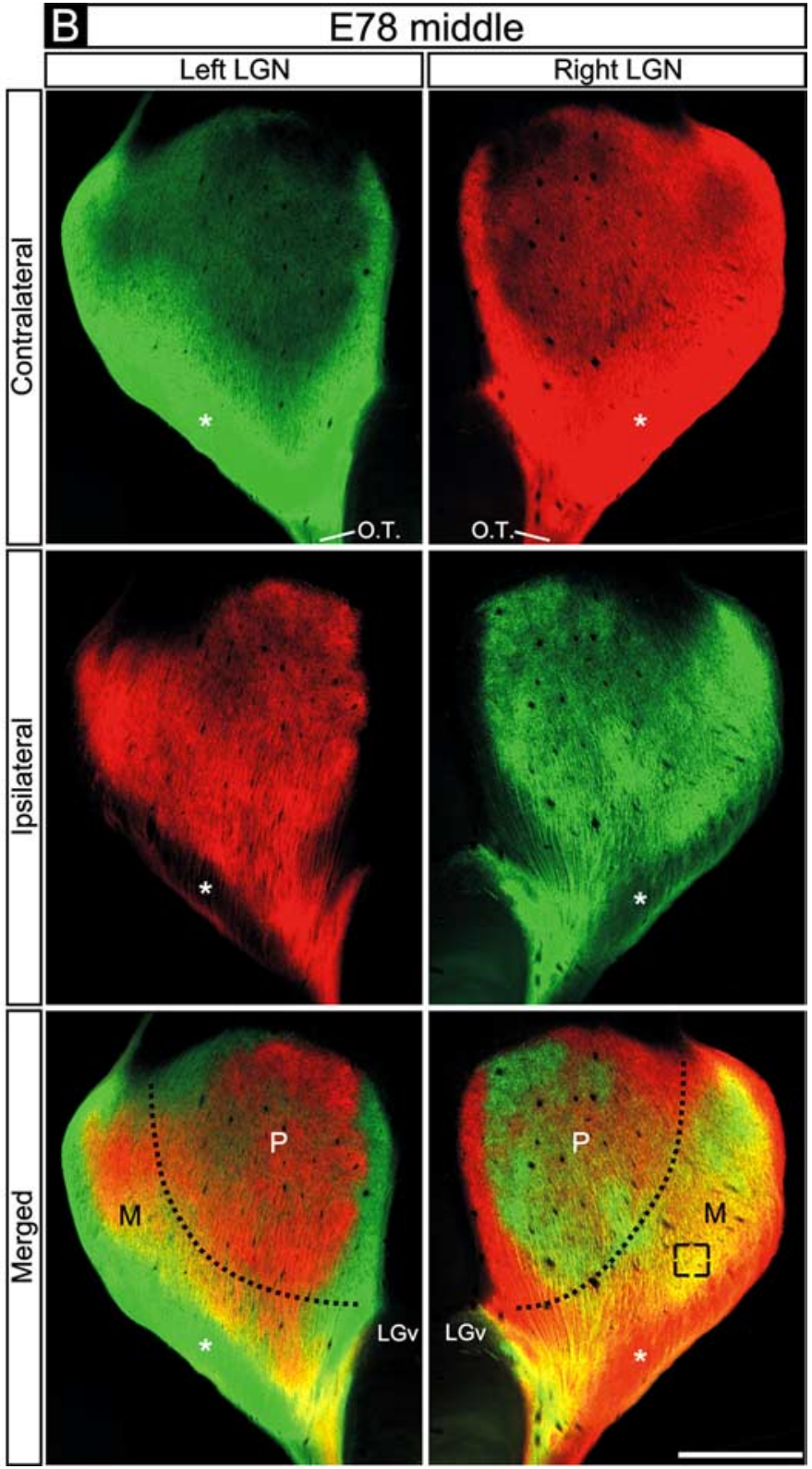

Kirkwood, 1984; Ziburkus et al., 2003; Ziburkus and Guido, 2005). In the present study, we sought to determine the scale at which axons from the two eyes intermingle within yellow "overlap" zones using high-magnification confocal imaging. Figure 4 shows confocal images from a portion of the $\mathrm{M}$ division of the E78 dLGN in which overlap appeared maximal (Fig. 3B, boxed region in bottom right panel). At this magnification, the red and green CT $\beta$ labels appear punctuate. Both the 7 - $\mu \mathrm{m}$-thick Z-plane optical stack (Fig. $4 A$ ) and the ultrathin, $0.3 \mu \mathrm{m}$ single optical section (Fig. $4 B$ ) reveal the presence of numerous cell body profiles (black regions) $\sim 5-10 \mu \mathrm{m}$ in diameter, each encompassed by both red and green puncta. In the ultrathin sections (Fig. $4 B$ ), both right and left eye axons can readily be seen in close proximity to individual cell bodies, and the two labels are present at approximately equal densities. Whether these profiles represent synaptic contacts onto single dLGN neurons or whether axons from the two eyes are segregated at the subcellular level could not be determined from this material. However, these data indicate that the overlap seen at relatively low 


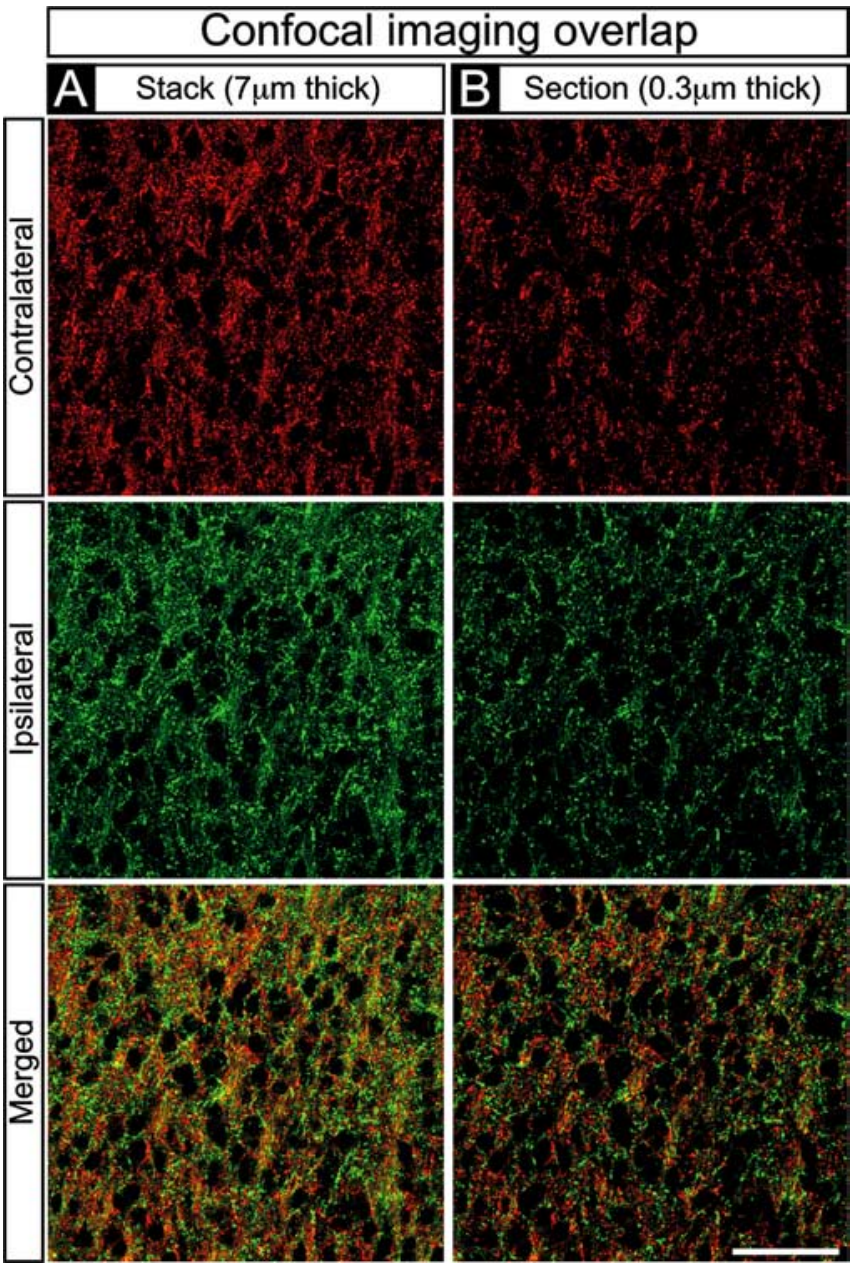

Figure 4. Confocal imaging of binocular overlap in the $\mathrm{dLGN} . \boldsymbol{A}, \boldsymbol{B}$, High-magnification confocal images of a region from the E78 $\mathrm{M}$ division dLGN in which overlap appeared maximal ("yellow" boxed region in Fig. 3B). Contralateral retinal axons (top rows), ipsilateral retinal axons (middle rows), and their merged representation (bottom rows) are shown. $A, A 7.0-\mu \mathrm{m}-$ thickZ-stack. $B, A 0.3-\mu \mathrm{m}$-thick single optical plane. $A, B$, Black oval profiles are dLGN cells. Red and green profiles appear punctate at high magnification, are present at approximately equal density, and surround every cell profile in the field of view, indicating that axons from the two eyes are intermingled at the level of single dLGN neurons. Scale bar, $50 \mu \mathrm{m}$.

magnifications indeed represents intermingling of axons from the two eyes on a scale comparable with that of a single dLGN neuron, consistent with the results of the physiological studies cited above (Shatz and Kirkwood, 1984; Ziburkus et al., 2003; Ziburkus and Guido, 2005).

\section{Retinogeniculate projections in the E84 macaque}

We next examined eye-specific projections using binocular CT $\beta$ tracing in an E83-E84 macaque embryo. Along the full rostrocaudal extent of the E84 dLGN and in both the M and P divisions, ganglion cell axons from the two eyes appeared segregated from one another. Right and left eye axons terminate in a complementary manner and exhibit a striking degree of mirror symmetry between the two hemispheres (Figs. $5 A, B, 6$ ). The only apparent source of colocalized signal from the two eyes arises from axon shafts coursing dorsally through the opposite-eye innervated layers in the ventral dLGN (Fig. 6). The high degree of eye-specific segregation and the mirror symmetry in the right and left dLGNs allows all six eye-specific domains to be reliably identified by this age (Fig. $5 A$, bottom row). In the caudal dLGN (Fig. $5 B$ ), the termination zones arising from each eye are less pronounced, preventing reliable identification of each eye-specific domain. These discontinuities are also present in the caudal aspect of the adult macaque dLGN (Hickey and Guillery, 1979). Nevertheless, a high degree of eye-specific segregation and symmetry is also present in the caudal region of the E84 dLGN (Fig. 5B, compare right with left dLGNs) (supplemental Fig. 3, available at www. jneurosci.org as supplemental material).

\section{Quantification of binocular overlap}

To quantify the degree of binocular overlap in the dLGN at the fetal ages described above, we performed a colocalization analysis of red-and green-labeled axons (see Materials and Methods). As shown in Figure 7, at E69, right and left eye axons intermingle throughout $\sim 90 \%$ of the dLGN. By E78, the degree of binocular overlap in the $\mathrm{P}$ division of the $\mathrm{dLGN}$ is $<10 \%$. The measured binocular overlap within the E78 M division is higher ( 35-60\% depending on rostrocaudal position). However, as mentioned above, fibers of passage likely contribute to the overlap signal quantified in the $\mathrm{M}$ division, and removing this source of artifact from the quantification analysis in an unbiased way is not feasible. By E84, eye-specific segregation is complete throughout all but the caudalmost part of the dLGN; quantified overlap is $\sim 2 \%$ in the rostral and middle dLGN and $\sim 8 \%$ in the caudal dLGN. We thus conclude that eye-specific segregation is initiated at least as early E69 and is virtually complete by E84.

\section{Discussion}

The principal findings of the present study are that eye-specific projections to the macaque dLGN are formed much earlier and more rapidly than previously reported and emerge from an initial state in which axons from the two eyes intermingle. The early emergence of eye-specific segregation defined here has important implications for identifying the mechanisms that control axon sorting in the developing visual system.

\section{Early and rapid eye-specific segregation in the dLGN}

In his classic study, Rakic (1976) used monocular injections of radioactive tracers and postinjection survival periods of $14 \mathrm{~d}$ to show that axons from the two eyes are intermingled on E78 before segregating into eye-specific domains by E124. This approach, which was a technical tour de force at the time, did not allow binocular overlap to be directly assessed; segregation had to be inferred by comparing the pattern of label in the two hemispheres. Moreover, radioactive tracers have a high potential for spillover in young tissues (LeVay et al., 1978), and, for this reason, the main conclusions of a number of early studies on ocular dominance column (ODC) development (LeVay et al., 1978; Shatz and Stryker, 1978; Stryker and Harris, 1986; Ruthazer et al., 1999) have recently been revised. Modern labeling techniques revealed that segregated ODCs are present at stages of development when radioactive tracers previously indicated they still overlapped (Crowley and Katz, 2000; Crair et al., 2001). Here we used sensitive tracers that do not have the potential for spillover to label retinogeniculate projections arising from both eyes. This allowed direct visualization and quantification of binocular projections to the dLGN. In the youngest embryo studied (E69), there was already pronounced segregation within the dorsalmost division of the rostral dLGN (Fig. $1 A, C$ ), and, throughout the E69 dLGN, there were clear regional biases in the density of inputs arising from one eye or the other $($ Fig. $1 B$ ). It could be that, at younger fetal ages, binocular overlap would be present throughout the entire dLGN, but successful dual-eye labeling is 

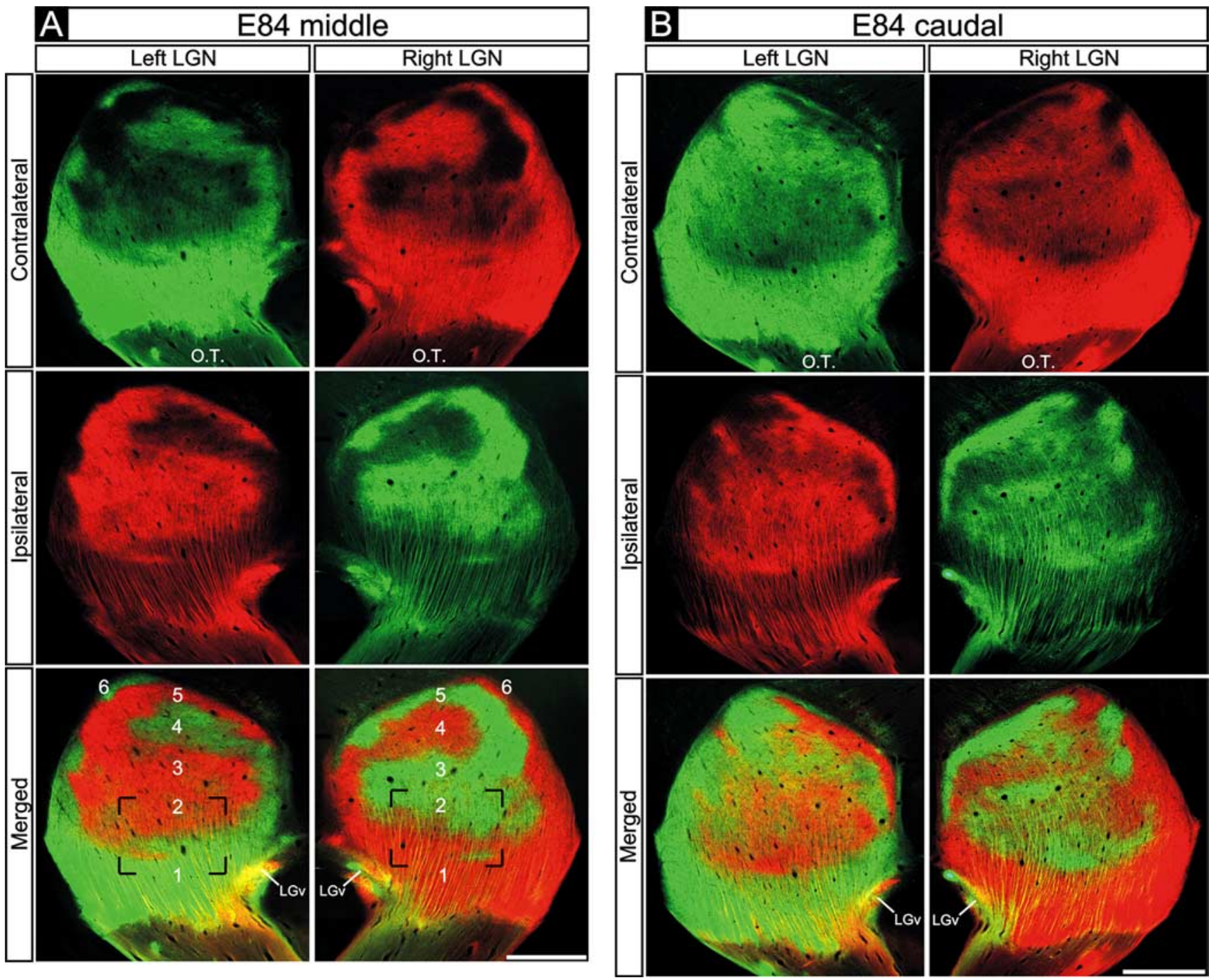

Figure 5. Retinogeniculate projections to the dLGN on E84. $A, B$, Contralateral retinal axons (top rows), ipsilateral retinal axons (middle rows), and their merged representation (bottom rows) in the right and left dLGNs of an E84 macaque. $A$, Middle dLGN. $B$, Caudal dLGN. $A, B$, Eye-specific segregation is present throughout the dLGN. $A$, All six eye-specific domains (1-6) are visible. $A, B$, Note the high degree of mirror symmetry in the pattern of eye-specific inputs in the two dLGNs. Coronal plane is shown. Dorsal is up. 0.T., Optic tract; LGv, ventral lateral geniculate nucleus. Scale bars, $550 \mu \mathrm{m}$.

exceedingly challenging in embryonic monkeys at ages younger than E69. It is also conceivable that some of the overlap we observed arises from fibers of passage or by late-arriving axons that target the koniocellular dLGN. It is notable that eye-specific segregation was apparent first in the dorsal aspect of the dLGN, in which fibers of passage are less numerous. Regardless of the fibers of passage issue, we show here that eye-specific segregation in the macaque is virtually completed by E84, more than 1 month earlier than previously described (Rakic 1976, 1977b), and occurs very rapidly, over a period of $\sim 15 \mathrm{~d}$.

\section{Regional differences in the appearance of eye-specific segregation in the dLGN}

On E69, segregation is already evident in the dorsalmost dLGN (Fig. 1C). The entire dLGN rotates during development such that this portion of the dLGN eventually resides in the caudalmost aspect and contains the foveal representation of the visual field (Rakic, 1977a). Eye-specific segregation may appear first in this portion of the nucleus because of maturational differences in foveal versus peripheral retina (La Vail et al., 1991) and/or be- cause fibers of passage are less numerous in the dorsal dLGN (ganglion cell axons course dorsally through the dLGN to reach their appropriate layer).

The issue of fibers of passage is difficult to resolve conclusively. The $\mathrm{P}$ anlagen is innervated by retinal fibers several weeks before the M segment (Meissirel et al., 1997). However, within the dLGN, M cells migrate into position before those in the $\mathrm{P}$ division (Rakic, 1977a; Shatz and Rakic, 1981). Thus, there does not appear to be an obvious link between the birth and migration of dLGN neurons with either the initial innervation or the subsequent segregation of ocular fibers in the $\mathrm{P}$ and $\mathrm{M}$ divisions. Only with the discovery of reliable markers for $\mathrm{M}$ versus $\mathrm{P}$ ganglion cell axons and their target cells could this issue could be addressed directly.

\section{Mechanisms underlying segregation of eye-specific inputs}

The predominant model of eye-specific segregation is centered on a Hebbian-type mechanism involving synapse turnover mediated by correlated retinal activity (for review, see Wong, 1999). Numerous observations support this model; in carnivores and 


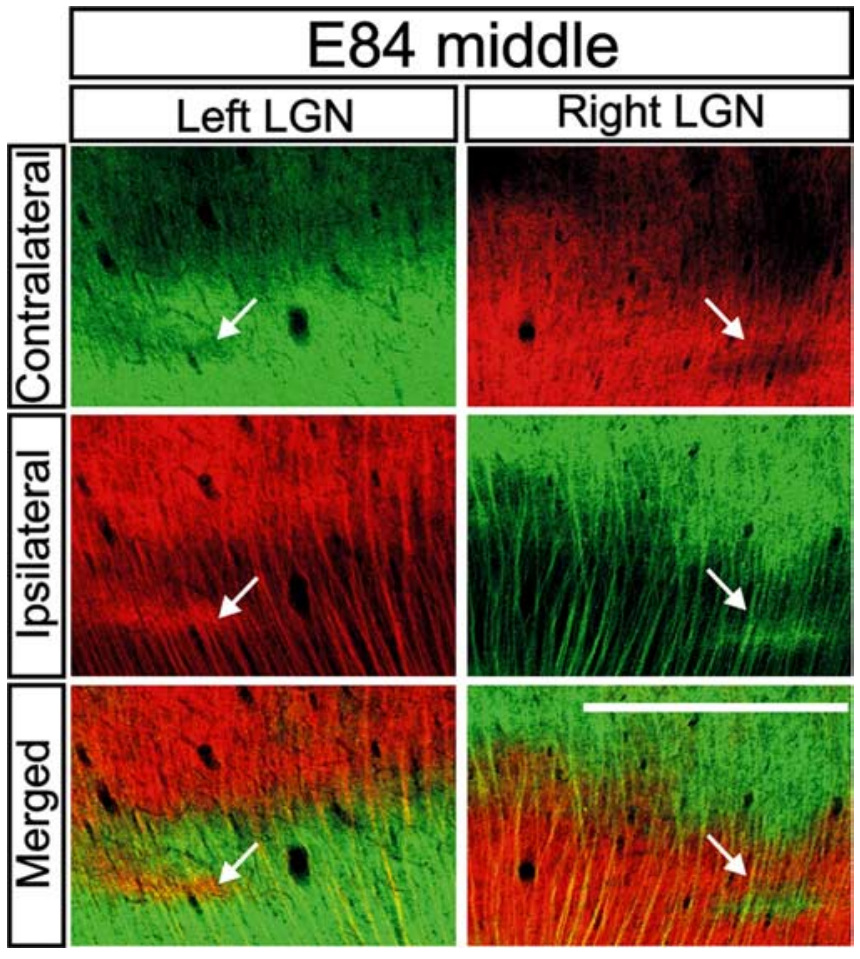

Figure 6. High degree of symmetry and segregation in E84 dLGN. Contralateral retinal axons (top row), ipsilateral retinal axons (middle row), and their merged representation (bottom row) in the right and left middle dLGNs of an E84 macaque (for orientation, see Fig. 5A, framed regions) are shown. Note the high degree of segregation and mirror symmetry in the pattern of eye-specific inputs in the two dLGNs, even for small features within retinal projections (compare arrows in right and left dLGNs). Coronal plane is shown. Dorsal is up. 0.T., Optic tract; LGV, ventral lateral geniculate nucleus. Scale bar, $550 \mu \mathrm{m}$.

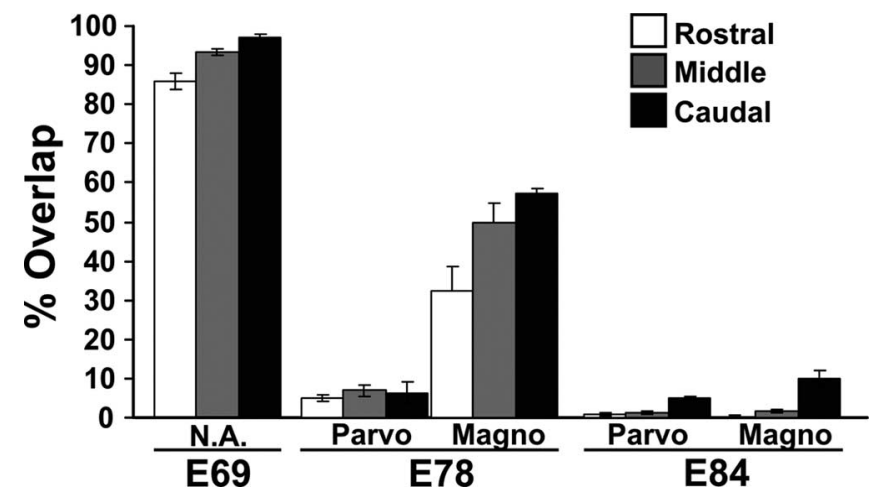

Figure 7. Quantification of binocular overlap in the developing macaque dLGN. The percentage of the dLGN containing axons from the right and left eyes across the rostral, middle, and caudal dLGN at E69, E78, and E84 is shown. Distinction is made between the degree the parvocellular (Parvo) versus magnocellular (Magno) dLGN on E78 and E84. (The M vs P regions cannot be reliably distinguished from all portions of the E69 $\mathrm{dLGN}$.) Serial sections through the right and left dLGN were quantified for each age ( $n=6-10$ sections per rostral, $6-10$ per middle, and $6-10$ per caudal, depending on the size of the dLGN).

rodents, functional retinogeniculate synapses are present during the binocular overlap period (Shatz and Kirkwood, 1984; Ziburkus et al., 2003; Ziburkus and Guido, 2005), and perturbing retinal activity alters eye-specific segregation (Penn et al., 1998; Chapman, 2000; Rossi et al., 2001; Huberman et al., 2002). However, segregated dLGN layers can emerge in the complete absence of binocular interactions (Williams et al., 1994), and, recently, the role of correlated retinal activity has been further challenged by the demonstration that eye-specific segregation occurs in the dLGN even when correlated retinal activity is eliminated (Huberman et al., 2003). Thus, the mechanisms underlying the eyespecific segregation process remain controversial (for review, see Chalupa and Huberman, 2004; Grubb and Thompson, 2004).

It is notable that, with few exceptions, studies in this field have been performed in mice or ferrets. These animal models offer clear advantages because they are born at a relatively immature stage of brain development. However, because of their relatively rapid development, manipulating retinal activity in these species undoubtedly impacts many of the cellular events that can influence targeting of ganglion cell axons in the dLGN, such as ganglion cell axon growth (Sretavan et al., 1988; Goldberg et al., 2002), neuronal migration (Rakic and Komuro, 1995), ganglion cell survival (Jeyarasasingam et al., 1998), and growth cone responses to axon guidance cues (Ming et al., 2001). Thus, it is still unclear which developmental processes underlie the formation of segregated retinogeniculate projections.

Having delimited the developmental period during which eye-specific retinogeniculate segregation occurs in the macaque, we compared this with other ontogenetic events in the monkey visual system (supplemental Fig. 4, available at www.jneurosci. org as supplemental material). In fetal monkey, retinogeniculate arbors undergo progressive growth beginning on $\sim$ E77 and until E140+ (Snider et al., 1999). Thus, eye-specific segregation parallels only the earliest stages of ganglion cell arbor formation. Also, whereas in the cat, pruning of axonal side branches coincides with eye segregation (Sretavan and Shatz, 1984) and is dependent on binocular competition (Garraghty et al., 1988), in the fetal monkey, retinogeniculate axons never exhibit side branches (Snider et al., 1999), and monocular enucleation does not influence the size of retinogeniculate arbors stemming from the remaining eye (Wefers et al., 2000).

Based on the temporal coincidence between eye-specific segregation as documented by autoradiography (Rakic, 1976, 1977b) and ganglion cell axon loss (Rakic and Riley, 1983), it was proposed that eye-specific segregation is attributable to the loss of ganglion cell axons projecting to inappropriate regions of the dLGN (Rakic, 1986). However, our data show that eye-specific segregation is complete more than 1 week before there is any evidence of ganglion cell axon loss (Rakic and Riley, 1983) (supplemental Fig. 4, available at www.jneurosci.org as supplemental material). Our results also indicate that eye-specific segregation in the macaque primarily precedes the major phase of synaptogenesis in the dLGN (Hendrickson and Rakic, 1977) (supplemental Fig. 4, available at www.jneurosci.org as supplemental material). Thus, it appears that ganglion cell apoptosis and synapse elimination are not directly involved in eye-specific segregation in the macaque.

Because axon pruning, synapse elimination, and ganglion cell death are unlikely to underlie the formation of eye-specific domains in the dLGN, what could account for this phenomenon? One possibility is that, shortly after ganglion cell axons arrive in the dLGN, axon guidance cues induce a rapid retargeting of ganglion axons to their appropriate eye-specific territories, wherein they undergo terminal arbor expansion. Such a mechanism has been proposed to underlie targeting of eye-specific dLGN projections and ODCs in ferrets (Crowley and Katz, 2000; Huberman et al., 2003), and afferent targeting through molecules such as ephrins is well documented in visual and other projection systems (Feldheim et al., 2000) (for review, see Bolz et al., 2004). Experiments are beginning to identify molecular markers of functional subdivisions of the carnivore and primate dLGN (Ka- 
wasaki et al., 2004), but whether these are directly involved in the targeting of ganglion cell axons still remains to be determined. Axon guidance cues that strictly demarcate or regulate the development of eye-specific territories remain to be discovered. The precise developmental period for eye-specific segregation documented here provides the necessary framework for future experiments aimed at identifying these cues.

\section{References}

Bolz J, Uziel D, Muhlfriedel S, Gullmar A, Peuckert C, Zarbalis K, Wurst W, Torii M, Levitt P (2004) Multiple roles of ephrins during the formation of thalamocortical projections: maps and more. J Neurobiol 59:82-94.

Brunso-Bechtold JK, Casagrande VA (1982) Early postnatal development of laminar characteristics in the dorsal lateral geniculate nucleus of the tree shrew. J Neurosci 2:589-597.

Brunso-Bechtold JK, Casagrande VA (1985) Presence of retinogeniculate fibers is essential for initiating the formation of each interlaminar space in the lateral geniculate nucleus. Brain Res 352:123-126.

Casagrande VA, Condo GJ (1988a) The effect of altered neuronal activity on the development of layers in the lateral geniculate nucleus. J Neurosci 8:395-416.

Casagrande VA, Condo GJ (1988b) Is binocular competition essential for layer formation in the lateral geniculate nucleus? Brain Behav Evol 31:198-208.

Chalupa LM, Huberman AD (2004) New perspectives on the role of neural activity in development of the visual system. In: The newest cognitive neurosciences, Ed 3. Cambridge, MA: MIT.

Chapman B (2000) Necessity for afferent activity to maintain eye-specific segregation in ferret lateral geniculate nucleus. Science 287:2479-2482.

Crair MC, Horton JC, Antonini A, Stryker MP (2001) Emergence of ocular dominance columns in cat visual cortex by 2 weeks of age. J Comp Neurol 430:235-249.

Crowley JC, Katz LC (2000) Early development of ocular dominance columns. Science 290:1321-1324.

Dehay C, Giroud P, Berland M, Smart I, Kennedy H (1993) Modulation of the cell cycle contributes to the parcellation of the primate visual cortex. Nature 366:464-466.

Feldheim DA, Kim YI, Bergemann AD, Frisen J, Barbacid M, Flanagan JG (2000) Genetic analysis of ephrin-A2 and ephrin-A5 shows their requirement in multiple aspects of retinocollicular mapping. Neuron 25:563-574.

Garraghty PE, Shatz CJ, Sretavan DW, Sur M (1988) Axon arbors of X and Y retinal ganglion cells are differentially affected by prenatal disruption of binocular inputs. Proc Natl Acad Sci USA 85:7361-7365.

Godement P, Salaun J, Imbert M (1984) Prenatal and postnatal development of retinogeniculate and retinocollicular projections in the mouse. J Comp Neurol 230:552-575.

Goldberg JL, Espinosa JS, Xu Y, Davidson N, Kovacs GT, Barres BA (2002) Retinal ganglion cells do not extend axons by default: promotion by neurotrophic signaling and electrical activity. Neuron 33:689-702.

Grubb MS, Thompson ID (2004) The influence of early experience on the development of sensory systems. Curr Opin Neurobiol 14:503-512.

Hendrickson A, Rakic P (1977) Histogenesis and synaptogenesis in the dorsal lateral geniculate nucleus (LGd) of the fetal monkey brain. Anat Rec 187:602.

Hickey TL, Guillery RW (1979) Variability of laminar patterns in the human lateral geniculate nucleus. J Comp Neurol 183:221-246.

Huberman AD, Stellwagen D, Chapman B (2002) Decoupling eye-specific segregation from lamination in the lateral geniculate nucleus. J Neurosci 22:9419-9429.

Huberman AD, Wang GY, Liets LC, Collins OA, Chapman B, Chalupa LM (2003) Eye-specific retinogeniculate segregation independent of normal neuronal activity. Science 300:994-998.

Huh GS, Boulanger LM, Du H, Riquelme PA, Brotz TM, Shatz CJ (2000) Functional requirement for class I MHC in CNS development and plasticity. Science 290:2155-2159.

Jeffrey G (1984) Retinal ganglion cell death and terminal field retraction in the developing rodent visual system. Brain Res 315:81-96.

Jeyarasasingam G, Snider CJ, Ratto GM, Chalupa LM (1998) Activityregulated cell death contributes to the formation of ON and OFF alpha ganglion cell mosaics. J Comp Neurol 394:335-343.
Jones EG (1985) The thalamus. New York: Plenum.

Kawasaki H, Crowley JC, Livesey FJ, Katz LC (2004) Molecular organization of the ferret visual thalamus. J Neurosci 24:9962-9970.

La Vail MM, Rapaport DH, Rakic P (1991) Cytogenesis in the monkey retina. J Comp Neurol 309:86-114.

LeVay SM, Stryker MP, Shatz CJ (1978) Ocular dominance columns and their development in layer IV of the cat's visual cortex: a quantitative study. J Comp Neurol 179:223-224.

Linden DC, Guillery RW, Cucchiaro J (1981) The dorsal lateral geniculate nucleus of the normal ferret and its postnatal development. J Comp Neurol 203:189-211.

Livingstone M, Hubel D (1988) Segregation of form, color, movement, and depth: anatomy, physiology, and perception. Science 240:740-749.

Meissirel C, Wikler KC, Chalupa LM, Rakic P (1997) Early divergence of magnocellular and parvocellular functional subsystems in the embryonic primate visual system. Proc Natl Acad Sci USA 94:5900-5905.

Ming G, Henley J, Tessier-Lavigne M, Song H, Poo M (2001) Electrical activity modulates growth cone guidance by diffusible factors. Neuron 29:441-452.

Muscat L, Huberman AD, Jordan CL, Morin LP (2003) Crossed and uncrossed retinal projections to the hamster circadian system. J Comp Neurol 466:513-524.

Penn AA, Riquelme PA, Feller MB, Shatz CJ (1998) Competition in re tinogeniculate patterning driven by spontaneous activity. Science 279:2108-2112.

Rakic P (1974) Neurons in rhesus monkey visual cortex: systematic relation between time of origin and eventual disposition. Science 183:425-427.

Rakic P (1976) Prenatal genesis of connections subserving ocular dominance in the rhesus monkey. Nature 261:467-471.

Rakic P (1977a) Genesis of the dorsal lateral geniculate nucleus in the rhesus monkey: site and time of origin, kinetics of proliferation, routes of migration and pattern of distribution of neurons. J Comp Neurol 176:23-52.

Rakic P (1977b) Prenatal development of the visual system in rhesus monkey. Philos Trans R Soc Lond B Biol Sci 278:245-602.

Rakic P (1986) Mechanism of ocular dominance segregation in the lateral geniculate nucleus: competitive elimination hypothesis. Trends Neurosci $8: 11-15$.

Rakic P, Komuro H (1995) The role of receptor/channel activity in neuronal cell migration. J Neurobiol 26:299-315.

Rakic P, Riley KP (1983) Regulation of axon number in primate optic nerve by prenatal binocular competition. Nature 305:135-137.

Rathjen FG, Gierer A (1981) Cholera-toxin binding to cells of developing chick retina analyzed by fluorescence-activated cell sorting. Brain Res 227:539-549.

Roonwal ML, Mohnot SM (1977) Primates of South Asia- ecology, sociobiology, and behavior. Cambridge, MA: Harvard UP.

Rossi FM, Pizzorusso T, Porciatti V, Marubio LM, Maffei L, Changuex JP (2001) Requirement of the nicotinic acetylcholine receptor $\beta 2$ subunit for the anatomical and functional development of the visual system. Proc Natl Acad Sci USA 98:6453-6458.

Ruthazer ES, Baker GE, Stryker MP (1999) Development and organization of ocular dominance bands in primary visual cortex of the sable ferret. J Comp Neurol 407:151-165.

ShatzCJ (1983) The prenatal development of the cats retinogeniculate pathway. J Neurosci 3:482-499.

Shatz CJ, Kirkwood PA (1984) Prenatal development of functional connections in the cat's retinogeniculate pathway. J Neurosci 4:1378-1397.

Shatz CJ, Rakic P (1981) The genesis of efferent connections from the visual cortex of the fetal rhesus monkey. J Comp Neurol 196:287-307.

Shatz CJ, Stryker MP (1978) Ocular dominance in layer IV of the cat's visual cortex and the effects of monocular deprivation. J Physiol (Lond) 281:267-283.

Smart I, Dehay C, Giroud P, Berland M, Kennedy H (2002) Unique morphological features of the proliferative and postmitotic compartments of the neural epithelium giving rise to striate and extrastriate cortex in the monkey. Cereb Cortex 12:37-53.

Snider CJ, Dehay C, Berland M, Kennedy H, Chalupa LM (1999) Prenatal development of retinogeniculate axons in the macaque monkey during segregation of binocular inputs. J Neurosci 19:220-228.

So KF, Schneider GE, Frost DO (1978) Postnatal development of retinal projections to the lateral geniculate body in Syrian hamsters. Brain Res 142:343-352. 
Sretavan D, Shatz CJ (1984) Prenatal development of individual retinogeniculate axons during the period of segregation. Nature 308:845-848.

Sretavan DW, Shatz CJ, Stryker MP (1988) Modification of retinal ganglion cell axon morphology by prenatal infusion of tetrodotoxin. Nature 336:468-471.

Stellwagen D, Shatz CJ (2002) An instructive role for retinal waves in the development of retinogeniculate connectivity. Neuron 33:357-367.

Stryker MP, Harris WA (1986) Binocular impulse blockade prevents the formation of ocular dominance columns in cat visual cortex. J Neurosci 6:2117-2133.

Wefers CJ, Dehay C, Berland M, Kennedy H, Chalupa LM (2000) Binocular competition does not regulate retinogeniculate arbor size in fetal monkey. J Comp Neurol 427:362-369.
Williams RW, Hogan D, Garraghty PE (1994) Target recognition and visual maps in the thalamus of achiasmatic dogs. Nature 367:637-639.

Wong RO (1999) Retinal waves and visual system development. Annu Rev Neurosci 22:29-47.

Wu CC, Russell RM, Karten HJ (1999) The transport rate of cholera toxin B subunit in the retinofugal pathways of the chick. Neuroscience 92:665-676.

Ziburkus J, Guido W (2005) Loss of binocular responses and reduced retinal convergence during the period of retinogeniculate axon segregation. J Neurophysiol, in press.

Ziburkus J, Lo FS, Guido W (2003) Nature of inhibitory postsynaptic activity in developing relay cells of the lateral geniculate nucleus. J Neurophysiol 90:1063-1070. 